APS

physics

This is the accepted manuscript made available via CHORUS. The article has been published as:

\title{
Method for the experimental measurement of bulk and shear loss angles in amorphous thin films
}

Gabriele Vajente, Mariana Fazio, Le Yang, Anchal Gupta, Alena Ananyeva, Garilynn Billinsley, and Carmen S. Menoni

Phys. Rev. D 101, 042004 - Published 24 February 2020

DOI: 10.1103/PhysRevD.101.042004 
${ }_{22}$ High precision optical metrology relies on high finesse and ${ }_{23}$ low loss optical resonant cavities, built with high reflec24 tivity dielectric mirrors. The ultimate limit to the length ${ }_{25}$ stability of such cavities is often determined by thermal ${ }_{26}$ motion of the cavity components. In many cases, such ${ }_{27}$ as in interferometric gravitational wave (GW) detectors 28 [1-4], the limit thermal noise comes from the Brownian 29 motion of the dielectric coatings deposited on the mirrors 30 [5], and composed of alternating layers of amorphous ox31 ides: silica and titania-doped tantala for the Advanced ${ }_{32} \mathrm{GW}$ detectors [6]. The amplitude of Brownian noise can 33 be linked to the material internal friction by use of the ${ }_{34}$ Fluctuation-Dissipation Theorem [7,8]. In the simplest ${ }_{35}$ possible approximation the energy lost per cycle due to ${ }_{36}$ internal friction is modeled as a fraction of the total elas${ }_{37}$ tic energy $E$ stored in one of the resonator eigenmodes, 38 using one single number usually called the loss angle $\phi$ :

$$
\langle\Delta E\rangle_{\text {cycle }}=\phi\langle E\rangle
$$

${ }_{39}$ If the surface of the mirror is probed with a Gaussian ${ }_{40}$ laser beam with beam radius $w$, then in the simple ap${ }_{41}$ proximation described above the displacement noise due 42 to Brownian motion has a power spectral density [9] given 43 by $[10]$

$$
S(f)=\frac{4 k_{B} T}{\pi^{2} f} \frac{\left(1+\nu_{S}\right)\left(1-2 \nu_{S}\right)}{Y_{S}} \frac{d}{w} \phi_{C}
$$

${ }_{44}$ where $f$ is the frequency, $k_{B}$ is Boltzmann's constant, $T$ ${ }_{45}$ the temperature, $Y_{S}$ and $\nu_{S}$ the Young's modulus and ${ }_{46}$ Poisson ratio of the mirror substrate, $d$ is the coating

\footnotetext{
*vajente@caltech.edu
}

${ }_{47}$ thickness and $\phi_{C}$ the coating average loss angle. In this ${ }_{48}$ model the beam is assumed to be much larger than the ${ }_{49}$ film thickness, and there is no distinction between energy ${ }_{50}$ lost in the shear and bulk deformations of the mirror.

${ }_{51}$ However, even for an amorphous material, the bulk and ${ }_{52}$ shear moduli are not equal, and therefore by extension ${ }_{53}$ there is no reason to assume that the bulk and shear loss ${ }_{54}$ angles have the same value. The theory of room tem${ }_{55}$ perature loss in amorphous materials $[11,12]$ ascribes ${ }_{56}$ the energy loss mechanism to the presence of two-level ${ }_{57}$ systems, effectively described as double-well potentials 58 with thermally excited tunneling between the two min59 ima. The material mechanical loss is determined by the ${ }_{60}$ density of the two-level systems, by the distribution of ${ }_{61}$ the potential wells and barriers, and by the coupling of ${ }_{62}$ the two-level systems to the macroscopic elastic strain. ${ }_{63}$ There is no reason to assume that the two-level systems 64 would couple in the same way to bulk and shear strains. ${ }_{65}$ Lacking a theoretical or phenomenological reason to as${ }_{66}$ sume the contrary, in computing the thermal noise due 67 to the elastic energy loss in a multilayer coating, one ${ }_{68}$ needs to take into account both shear and bulk deforma${ }_{69}$ tions and allow for the loss mechanisms to be different. ${ }_{70}$ The resulting displacement noise depends on the value ${ }_{71}$ of both bulk and shear loss angles in a way more com72 plex than what shown in equation 2 [13]. In particular, ${ }_{73}$ it is generally believed that the shear loss angle is more ${ }_{74}$ relevant than the bulk loss angle, when the beam size is ${ }_{75}$ comparable with the film thickness. Therefore, to have 76 an accurate estimate of the Brownian noise in an optical 77 system, it is important to have a reliable measurement 78 of both loss angles.

${ }_{79}$ The most common technique to measure the loss an${ }_{80}$ gle(s) of a thin film is to deposit it on a high quality ${ }_{81}$ resonator, and measure the decay time $\tau$ of a subset of ${ }_{82}$ the eigenmodes. This can be accomplished by exciting 83 the resonator and tracking the oscillation amplitude of 
${ }_{84}$ each mode over time:

$$
A_{i}(t)=A_{0} e^{-t / \tau_{i}}
$$

${ }_{85}$ Some excess energy loss is always present for all modes, ${ }_{86}$ due for example to contact at the suspension point or ${ }_{87}$ substrate clamp. It is generally possible to find a suitable 88 set of eigenmodes for which recoil losses are negligible, 89 and are well decoupled from the environment. Typically 90 those modes allow probing the material loss angle over ${ }_{91}$ a sufficiently large range of frequencies. Measuring the ${ }_{92}$ decay time of this set of eigenmodes allows probing the ${ }_{93}$ value and frequency dependency of the loss angles. For ${ }_{94}$ each eigenmode at a frequency $f_{i}$, the decay time $\tau_{i}$ is ${ }_{95}$ linked to the coated resonator quality factor $Q_{i}$ and loss ${ }_{96}$ angle $\phi_{i}$ by the following relations

$$
\phi_{i}=\frac{1}{Q_{i}}=\frac{1}{\pi f_{i} \tau_{i}}
$$

${ }_{97}$ The loss angle $\phi_{i}$ of the coated resonator should not be ${ }_{98}$ confused with the loss angle of the materials. It is related 99 to the total elastic energy loss per cycle, and we can 100 therefore divide it in two terms: a contribution coming 101 from the substrate $\phi_{i}^{(s u b)}$ and a contribution coming from ${ }_{102}$ the thin film $\phi_{i}^{(f i l m)}$. The contribution of each term to ${ }_{103}$ the total loss angle is weighted by the amount of elastic ${ }_{104}$ energy which is stored in the substrate and in the film, 105 on average:

$$
\begin{aligned}
\phi_{i}^{(\text {coated })} & =\frac{E_{i}^{(\mathrm{sub})} \phi_{i}^{(\mathrm{sub})}+E_{i}^{(\mathrm{film})} \phi_{i}^{(\mathrm{film})}}{E_{i}^{(\mathrm{sub})}+E_{i}^{(\mathrm{film})}} \\
& =\left(1-D_{i}\right) \phi_{i}^{(\mathrm{sub})}+D_{i} \phi_{i}^{(\mathrm{film})}
\end{aligned}
$$

106 where we have introduced the mode dependent dilution 107 factor $D_{i}=E_{i}^{(\text {film })} / E_{i}^{(\text {tot })}$. The substrate loss angle can 108 be measured before any film is deposited, and it is usually 109 assumed to remain unchanged by the deposition process. ${ }_{110}$ Therefore the difference of loss angles as measured before 111 and after the film is deposited can be used to extract the ${ }_{112}$ loss angle of the material composing the film. We define 113 the excess loss of the coated sample as

$$
\delta \phi_{i}=\phi_{i}^{\text {(coated) }}-\left(1-D_{i}\right) \phi_{i}^{\text {(sub) }}=D_{i} \phi_{i}^{\text {(film) }}
$$

${ }_{114}$ The dilution factors $D_{i}$ can be computed using finite el115 ement simulations of the resonators, knowing the elastic 116 properties of the material, or extracted directly from the ${ }_{117}$ change in the eigenmode resonant frequencies [14]. Since 118 we are interested in measuring the bulk and shear loss 119 angles $\phi_{B, i}$ and $\phi_{S, i}$, we need to modify the model in ${ }_{120}$ equation 6 above as follows

$$
\delta \phi_{i}=D_{B, i} \phi_{B, i}+D_{S, i} \phi_{S, i}
$$

121 where we defined the new bulk and shear dilution factors 122 as $D_{B, i}=E_{B, i}^{\text {(film) }} / E_{i}^{\text {(tot) }}$ and $D_{S, i}=E_{S, i}^{\text {(film) }} / E_{i}^{\text {(tot) }}$, so ${ }_{123}$ that $D_{i}=D_{B, i}+D_{S, i}$. Below we will describe how the 124 elastic properties can be extracted from the modal fre125 quencies and then used to calculate the dilution factors 126 using a finite element model.
${ }_{127}$ In this paper we describe how it is possible to analyze 128 the resonant mode decay times of a thin film deposited 129 on a silica disk-shaped substrate measured in a Gentle ${ }_{130}$ Nodal Suspension [15, 16], and express the film proper${ }_{131}$ ties in terms of bulk and shear loss angle. In summary 132 the analysis proceed in several steps. First of all, the ${ }_{133}$ elastic properties of the film are extracted from the shift 134 in the resonator eigenmodes due to the addition of the ${ }_{135} \mathrm{film}$. This estimate is carried out with a Bayesian infer${ }_{136}$ ence analysis and includes uncertainties that model the ${ }_{137}$ limited knowledge and possible evolution with heat treat138 ment of the film density and thickness. More details on ${ }_{139}$ this first step in section II. The posterior probability dis140 tribution of the elastic properties are then used as priors ${ }_{141}$ for another Bayesian inference analysis, where the mea${ }_{142}$ sured excess losses introduced in equation 6 or equation ${ }_{143} 7$ are estimated based on a model of the material loss 144 angle(s). This procedure factor into the posterior distri145 bution of the loss angle the uncertainties in the material ${ }_{146}$ properties and possible correlation between the model ${ }_{147}$ parameters. More details in section III.

${ }_{148}$ Analysis of measurements in terms of bulk and shear loss 149 angles were done in the past for films on a cantilever com150 posed of alternating layers of silicon nitride and silica [17], 151 and for a titania-doped tantala film on a disk suspended 152 with a fiber [18].

${ }_{153}$ We show the result of our analysis for a titania-doped 154 tantala film as an example, and discuss how the experi155 mental data favor a model with different bulk and shear 156 loss angle over a simpler model with equal loss angles. ${ }_{157}$ The material studied here is comparable to what was con158 sidered in [18], and we note that the results we obtain are 159 different from those obtained in the previous work. More 160 on this topic in section III. Finally, in section IV we dis${ }_{161}$ cuss how the measured loss angles impact the estimate of 162 thermal noise for the Advanced LIGO gravitational wave 163 detector.

164

\section{MEASUREMENTS}

${ }_{165}$ The substrates used in this work consist of fused silica ${ }_{166}$ disks, $75 \mathrm{~mm}$ in diameter and $1 \mathrm{~mm}$ thick, supported at 167 the center by a gentle nodal suspension $[15,16]$. All the ${ }_{168}$ disk eigenmodes that have null deformation at the disk 169 center are accessible in this system, and have very low 170 recoil losses $\left(Q^{(\mathrm{sub})} \gtrsim 10^{8}\right)$. The largest fraction of elas171 tic energy is stored in shear deformation, but depending 172 on the mode shape, in particular on the number of ra173 dial nodes, there are non negligible amounts of energy in 174 the bulk deformation, allowing us to disentangle the two 175 contributions.

${ }_{176}$ The gentle nodal suspension allows simultaneous track177 ing of all modes, providing a measurement of both the 178 frequency and the decay time of each mode. All sub179 strates are characterized prior to coating, to measure the 180 substrate loss angles $\phi_{i}^{\text {(sub) }}$ and the frequency of each ${ }_{181}$ mode. A 270-nm-thick film of titania-doped tantala (27\% 182 cation concentration of titania) was then deposited with 


\begin{tabular}{|c|c|c|c|}
\hline & As deposited & Annealed $500^{\circ} \mathrm{C}$ & Annealed $600^{\circ} \mathrm{C}$ \\
\hline $\begin{array}{l}\text { Young's modulus } Y[\mathrm{GPa}] \\
\text { Poisson ratio } \nu\end{array}$ & $\begin{array}{c}118 \pm 3 \\
0.396 \pm 0.016\end{array}$ & $\begin{array}{c}120 \pm 3 \\
0.407 \pm 0.013\end{array}$ & $\begin{array}{c}128 \pm 4 \\
0.346 \pm 0.019\end{array}$ \\
\hline $\begin{array}{l}\text { Cation concentration } \\
\text { Thickness } t[\mathrm{~nm}] \\
\text { Density } \rho\left[\mathrm{kg} / \mathrm{m}^{3}\right]\end{array}$ & \multicolumn{3}{|c|}{$\begin{array}{c}73 \% \mathrm{Ta}, 27 \% \mathrm{Ti} \\
268 \pm 13 \\
6640 \pm 300\end{array}$} \\
\hline
\end{tabular}

TABLE I. Measured and estimated parameters of the titania-doped tantala thin film studied in this work. The thickness was measured on the as deposited samples, and the density estimated from the composition. The film elastic properties come from fits to the resonant mode data, as explained in the text. The uncertainties in thickness and density account for possible variations upon annealing, as explained in the main text.

183 ion beam sputtering on one face of the substrates. The 184 coated samples were then measured again, to obtain a 185 new set of mode frequencies and decay times. The sam186 ples were then subjected to a heat treatment (annealing), ${ }_{187}$ consisting of a slow ramp up to a target temperature, 188 hold for ten hours, and then a slow ramp down to room 189 temperature. The samples measured for this work have 190 been annealed at 500,600 and $700^{\circ} \mathrm{C}$. The film annealed 191 at $700^{\circ} \mathrm{C}$ showed signs of micro-crystallization, and there${ }_{192}$ fore the corresponding results are not considered in this 193 work. Ring downs were measured after each heat treat194 ment step, resulting in a set of excess loss angles $\left\{\delta \phi_{i}\right\}$ ${ }_{195}$ for the as-deposited samples and the annealed samples.

${ }_{196}$ The film thickness $t$ was measured with ellipsometry, and ${ }_{197}$ the relative concentration of titania and tantala was es198 timated from the measured refractive index and X-ray 199 photoelectron spectroscopy. The material density $\rho$ was 200 estimated with a linear interpolation between the two 201 oxide component densities, weighted with the measured 202 oxide concentration.

${ }_{203}$ The thin film changes the flexural rigidity of the disk, 204 resulting in a shift of all resonant mode frequencies. The 205 relative difference between the coated and uncoated disk 206 frequencies is roughly constant between 1 and $30 \mathrm{KHz}, 2$ 207 and equal to about $300 \mathrm{ppm}$, with variation between 208 modes of the order of $10-30 \mathrm{ppm}$, related to the film ${ }_{209}$ Poisson ratio. We used a finite element analysis (FEA) 210 carried out in COMSOL to find the values of the film 211 material Young's modulus $Y$ and Poisson ratio $\nu$ that 212 best reproduce the measured changes in resonant fre${ }_{213}$ quencies [6]. Instead of using directly COMSOL in the fit ${ }_{214}$ procedure, we first produced a random sampling of the 215 film properties space $[Y, \nu, t, \rho]$ and run a FEA for each 216 point. We then fit a third order polynomial function of ${ }_{217} Y, \nu, t$ and $\rho$ to the simulated frequency shifts, obtaining 218 a fast semi-analytical model that is accurate within tens 219 of $\mathrm{mHz}$. Using this fast model, we carried out a Bayesian 220 inference analysis [22] to estimate the probability distri221 bution and the confidence intervals for $Y$ and $\nu$. Table ${ }_{222}$ I summarizes all the measured parameters of the thin ${ }_{223}$ films. The results are dependent on the thickness and ${ }_{224}$ density of the film. The reader unfamiliar with Bayesian 225 inference analysis can refer for example to [22-24] for an 226 introduction. In section III we also describe the basics 227 of Bayesian inference, focusing on the application to the 228 extraction of bulk and shear loss angles from the mea229 surements.
${ }_{230}$ In this analysis we assumed that thickness and density 231 are constant, since we do not have yet a measurement of 232 how those film properties change with annealing. This as233 sumption is likely wrong, since changes of density, thick${ }_{234}$ ness and refractive index have been observed for other 235 amorphous materials $[6,25,26]$. However, we note that 236 the estimate of $Y$ and $\nu$ depends mostly on the product ${ }_{237}$ of thickness and density, that is, the surface density of 238 the material. Therefore, even though density and thick239 ness could each vary, if the annealing does not cause any 240 loss of material from the film, we expect that the prod241 uct of density and thickness will remain constant and 242 the estimate of the Young's modulus and Poisson ratio 243 to be correct. Nevertheless, in the analysis we accounted 244 for possible untracked changes by allowing a $\pm 5 \%$ un${ }_{245}$ certainty in the measured values for both thickness and 246 density.

${ }_{247}$ Two samples were coated with nominally equal materials 248 and deposition procedure. The two samples have been 249 measured separately, and the results collated together in 250 all computations.

\section{LOSS ANGLE ANALYSIS}

252 The main goal of this work is to determine which mate253 rial loss angle(s) model describes better the experimental 254 data points. For each set of measurements (as deposited 255 samples or annealed samples), we model the excess loss 256 angle assuming either equal or different bulk and shear ${ }_{257}$ loss angles for the film material. For both model choices, 258 we allow for a frequency dependency of the loss angles, 259 in the form of a power law or a linear relationship:

$$
\begin{aligned}
\phi_{\text {powerlaw }}\left(f ; \phi_{1}, \alpha\right) & =\phi_{1}\left(\frac{f}{1 \mathrm{kHz}}\right)^{\alpha} \\
\phi_{\text {linear }}\left(f ; \phi_{1}, m\right) & =\phi_{1}\left(1+m \frac{f-1 \mathrm{kHz}}{1 \mathrm{kHz}}\right)
\end{aligned}
$$

260 where $\phi_{1}$ is the loss angle at $1 \mathrm{kHz}, \alpha$ is the exponent of 261 the power law, and $m$ the slope of the linear relationship. ${ }_{262}$ The excess loss angles measured experimentally are then 263 modeled either with one loss angle, or with different bulk 264 and shear loss angles:

$$
\begin{aligned}
\delta \phi_{i}= & D_{i} \phi_{x}\left(f_{i} ; \phi_{1}, m\right) \\
\delta \phi_{i}= & D_{B, i} \phi_{x}\left(f_{i} ; \phi_{1, B}, m_{B}\right) \\
& +D_{S, i} \phi_{x}\left(f_{i} ; \phi_{1, S}, m_{S}\right)
\end{aligned}
$$



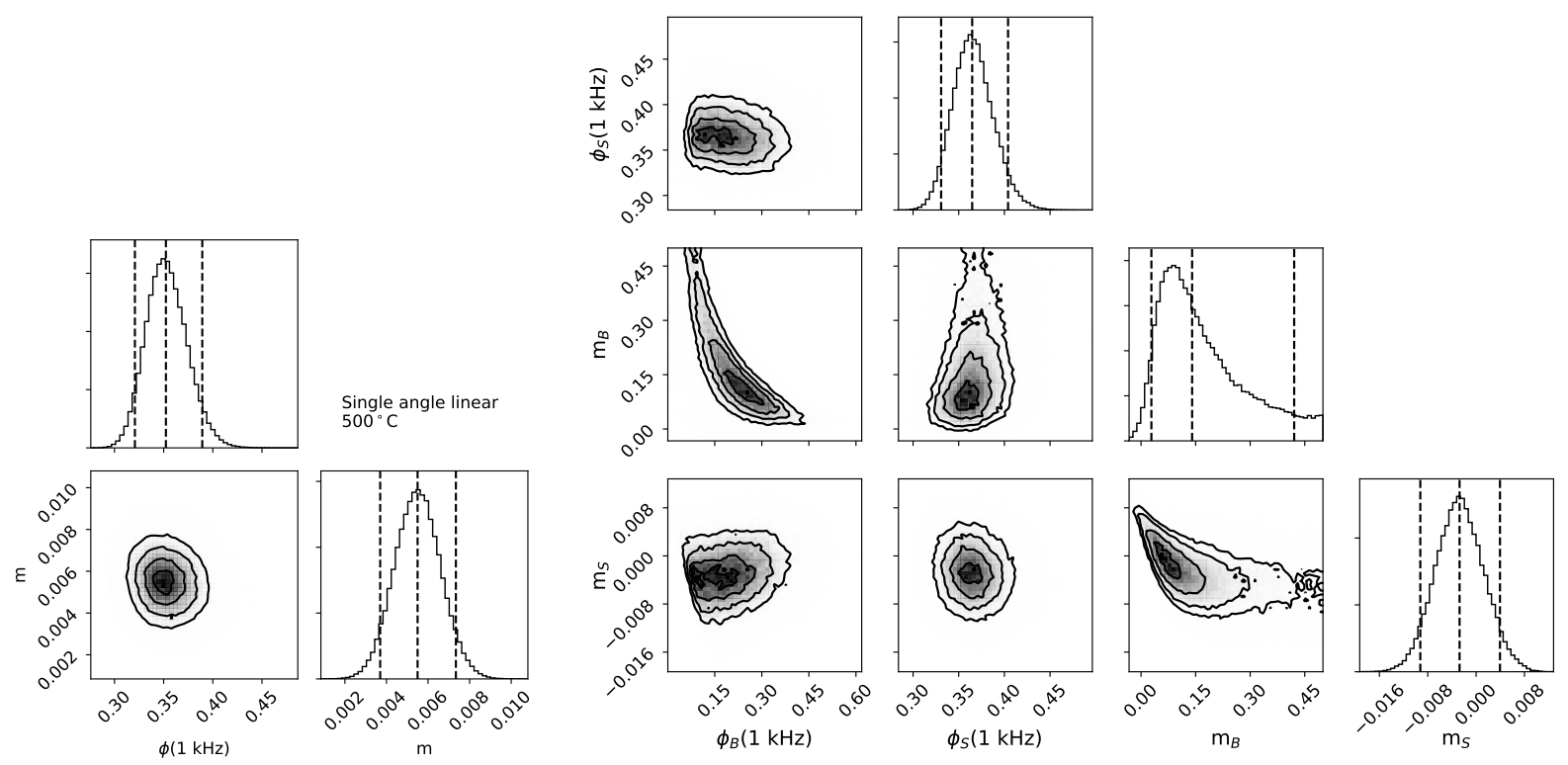

FIG. 1. Posterior probability distributions of the parameters of two loss models (left, one loss angle with linear frequency dependency; right, bulk and shear different loss angles with linear frequency dependency. The results shown here as an example, correspond to the measurements of titania-doped tantala films after annealing at $500^{\circ} \mathrm{C}$. The posterior probability distributions have been marginalized over the Young's modulus, the Poisson ratio, the film thickness and density.

\begin{tabular}{|c|c|c|c|c|}
\hline Model 1 & Model 2 & As deposited & Annealed 500 $^{\circ} \mathbf{C}$ & Annealed 600 $^{\circ} \mathbf{C}^{-1}$ \\
\hline Model 1 & Model 2 & As deposited & Annealed $500^{\circ} \mathrm{C}$ & Annealed $600^{\circ} \mathrm{C}$ \\
Single angle, power law & Bulk/Shear linear & -15.5 & -6.2 & -18.1 \\
Single angle, linear & Bulk/Shear linear & -7.4 & -1.6 & -10.1 \\
Bulk/Shear power law & Bulk/Shear linear & -0.6 & -0.2 & -1.8 \\
Single angle, power law & Bulk/Shear power law & -14.9 & -6.1 & -16.3 \\
Single angle, linear & Bulk/Shear power law & -6.8 & -1.4 & -8.3 \\
\hline
\end{tabular}

TABLE II. Bayesian odd ratios of the models considered in this analysis. Every table entry shows the logarithm of the bayesian ratio of Model 2 over Model 1. Negative values means that the data favors Model 2. The bulk-shear angle, linear-frequency dependency is favored for all annealing temperatures.

265 where $x$ can refer either to the linear or the power law 266 relation, for a total of four different models that could 267 describe the data: single loss angle with linear frequency ${ }_{268}$ dependency, single loss angle with power law frequency 269 dependency, bulk and shear loss angles with linear fre270 quency dependency, and bulk and shear loss angles with 271 power law frequency dependency. To quantitatively de272 termine which one of those four models better fits the 273 measured data, we follow a Bayesian approach, which ${ }_{274}$ provides us with the probability distribution of the pa275 rameters for each model, and also the relative probabil276 ity of the models, given the measured data set. In this 277 section we briefly outline the basics of the Bayesian ap278 proach, with particular emphasis to its application to the 279 problem at hand. The reader unfamiliar with Bayesian 280 inference analysis should refer, for example, to [22-24]
281 for a more detailed description.

${ }_{282}$ For each model, we want to compute the probability dis${ }_{283}$ tribution $\mathcal{P}\left(\theta \mid \mathcal{M}_{j}, \delta \phi_{i}\right)$ of the parameters $\theta$ (for example $284\left\{\phi_{1}, \alpha\right\}$ in the case of the single loss angle, power law 285 model) given the measured data $\left\{\delta \phi_{i}\right\}$ and assuming one 286 of the models, $\mathcal{M}_{j}$, to be valid. This probability distri287 bution is usually called the posterior distribution of the 288 model parameters. To compute it, we use Bayes' theorem $289[22]:$

$$
\mathcal{P}\left(\theta \mid \mathcal{M}_{j}, \delta \phi_{i}\right)=\frac{\mathcal{P}\left(\delta \phi_{i} \mid \mathcal{M}_{j}, \theta\right) \cdot \mathcal{P}\left(\theta \mid \mathcal{M}_{j}\right)}{\mathcal{P}\left(\delta \phi_{i} \mid \mathcal{M}_{j}\right)}
$$

290 where the term $\mathcal{P}\left(\delta \phi_{i} \mid \mathcal{M}_{j}, \theta\right)$ describes the probability 291 (likelihood) of obtaining the measured data given the 292 model and a specific value of the parameters, and the 

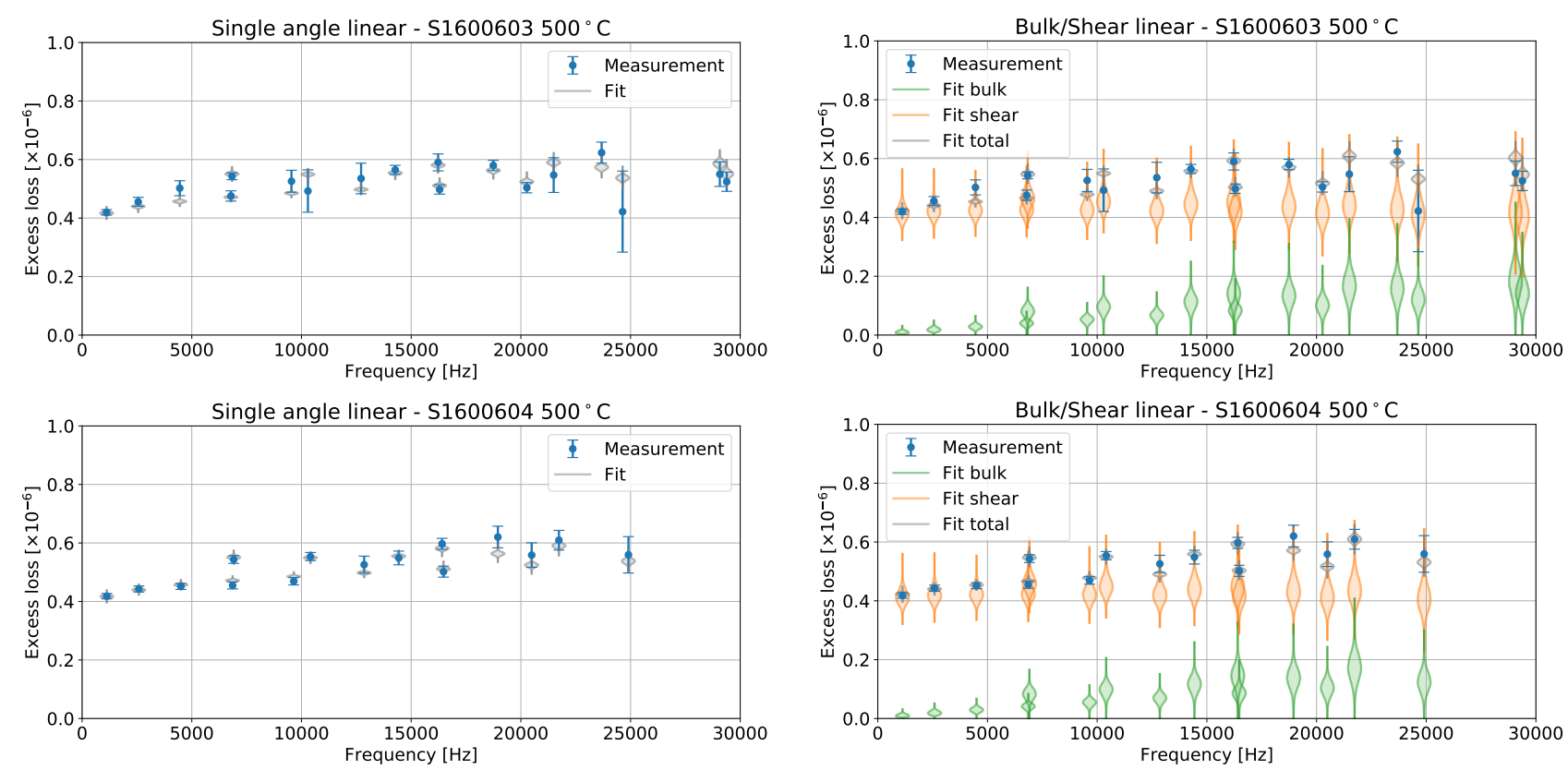

FIG. 2. Comparison of the measured and predicted excess loss angle (not the material loss angle) for the two samples, named S1600603 and S1600604, and shown respectively in the top and bottom rows. The results shown here correspond to the samples measured after annealing at $500^{\circ} \mathrm{C}$. The left column shows in grey the distribution of the excess loss angle for the single loss angle model. The right column instead shows the distributions for the bulk and shear loss angle model: in green the bulk contribution, in orange the shear contribution in grey the sum of the two. In both columns, the error bars data points represent the measured values. The violin plots instead represent the distribution of the predicted values, given the result of the Bayesian analysis.

\begin{tabular}{|c|c|c|c|c|}
\hline Heat treatment & $\begin{array}{c}\text { Bulk loss at 1 kHz } \\
\phi_{1, B}\left[10^{-3}\right]\end{array}$ & $\begin{array}{c}\text { Bulk loss slope } \\
m_{B}\end{array}$ & $\begin{array}{c}\text { Shear loss at 1 kHz } \\
\phi_{1, S}\left[10^{-3}\right]\end{array}$ & $\begin{array}{c}\text { Shear loss slope } \\
m_{S}\end{array}$ \\
\hline $30^{\circ} \mathrm{C}$ & $0.19 \pm 0.15$ & $0.24 \pm 0.19$ & $0.72 \pm 0.07$ & $-0.005 \pm 0.004$ \\
$500^{\circ} \mathrm{C}$ & $0.20 \pm 0.14$ & $0.14 \pm 0.20$ & $0.37 \pm 0.04$ & $-0.003 \pm 0.007$ \\
$600^{\circ} \mathrm{C}$ & $0.31 \pm 0.11$ & $0.09 \pm 0.07$ & $0.26 \pm 0.03$ & $-0.012 \pm 0.007$ \\
\hline
\end{tabular}

TABLE III. Parameters for the best fit to the data in terms of bulk and shear loss angles, with a linear dependency on frequency. The values quoted are the median of the probability distribution of each parameter given the data, and the $90 \%$ confidence intervals.

${ }_{293}$ term $\mathcal{P}\left(\theta \mid \mathcal{M}_{j}\right)$, usually called the prior probability dis294 tribution of the parameters, encodes our knowledge of the 295 possible values of the parameters, given a specific model, 296 before any measurement is taken. Finally, the term at the 297 denominator $\mathcal{P}\left(\delta \phi_{i} \mid \mathcal{M}_{j}\right)$ is the probability of obtaining 298 the measured data if the model is assumed, and allowing 299 any value for the parameter. This last term can be com300 puted as a normalization, by integrating the left hand 301 side of equation 12 over all values of $\theta$ and requiring the 302 result to be equal to one, since it is a probability distri303 bution. This term will play a role in the later selection 304 of the most likely model.

305 In our case, the data consist of the measured excess loss 306 angle $\delta \phi_{i}$ for both the samples measured, for each of the 307 accessible resonant mode frequencies, with the measure308 ment uncertainties. For any of the models, the data like${ }_{309}$ lihood $\mathcal{P}\left(\delta \phi_{i} \mid \mathcal{M}_{j}, \theta\right)$ is modeled as a normal distribution, 310 where each data point is an independent random variable 311 with variance given by the experimental uncertainties in
312 the measured quality factors. For each model, the pa313 rameter set $\theta$ is composed of two parts. First, we al314 low the film properties to vary within the uncertainties 315 described in section II: the Young's modulus and Pois316 son ratio have normal probability distributions centered 317 on the best fit of the resonant mode frequency shifts, 318 with variance given also by the fit, as reported in table $319 \mathrm{I}$; the coating density and thickness are also allowed to 320 vary with a normal probability distribution centered on 321 the nominal value and with a variance corresponding to 322 a $5 \%$ uncertainty as explained in section II. Secondly, 323 the prior distributions of the other model parameters are 324 assumed to be flat: the loss angle at $1 \mathrm{kHz}$ can vary 325 in the range $\phi_{1} \in\left[0,3 \times 10^{-3}\right]$ for all models; for the 326 power law loss angle models the exponent can vary in 327 the range $m \in[-2,2]$, while for the linear models the 328 slope is restricted to values that exclude negative loss 329 angles $m \in[-0.033,0.5]$. As we shall see, the results 330 are not very sensitive to the choice for the allowed range 

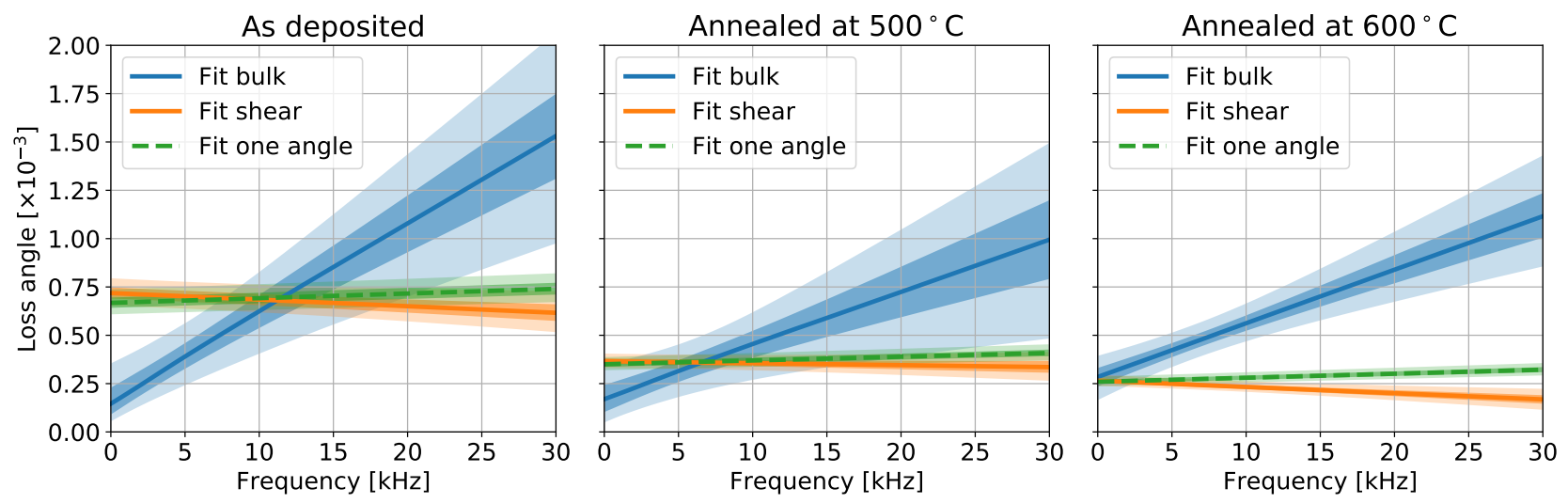

FIG. 3. Estimated loss angles as a function of frequency for the measured titania-doped tantala film, after each heat treatment step. In each panel, blue and orange shows the best fit to bulk and shear loss angles respectively, while the green dashed line correspond to the best fit to a single loss angle model.

331 of the parameters, meaning that the measured data is 332 increasing our knowledge of the models, as expected.

${ }_{333}$ There are many ways to use equation 12 to compute ${ }_{334}$ the posterior distribution of the model parameters. The 335 method most commonly used, and also adopted for this 336 work, is to numerically sample the posterior distribution, ${ }_{337}$ or in other words to compute a large set of points in 338 the parameter space, distributed in a way that follows 339 the posterior distribution. We carried out this sampling 340 using a Markov Chain Montecarlo (MCMC) algorithm 341 implemented with the Python package emcee [27]. The 342 results can then be used to numerically evaluate the dis343 tribution of each parameter. Since the model parameter 344 space is high dimensional, it is impossible to represent 345 graphically the full distribution. We therefore plot the 346 sets of all joint distributions of pairs of parameters. The ${ }_{347}$ results are shown in figure 1 for the two samples annealed 348 at $500^{\circ} \mathrm{C}$, and considering the following two models: one 349 single loss angle with linear frequency dependency, or 350 bulk and shear different loss angles with linear frequency 351 dependencies (similar results are available for all anneal352 ing temperatures and the power law models, but they 353 are not shown here for brevity). Each panel in the two 354 corner plots show the joint probability distribution for 355 pairs of parameters, as well as the probability distribu356 tion of each parameter, at the top of each column. Each ${ }_{357}$ of the contour plots in figure 1 represents the probability 358 distribution of the two parameters, given the data and as359 suming one of the models. All the other parameters are 360 allowed to take any value, a procedure often referred as 361 marginalization. The one-dimensional histograms show 362 the probability distribution of each parameter, marginal363 ized over all the others. The dashed lines represent the $36490 \%$ confidence intervals and the median of the poste365 rior distributions. Those values can be taken as the best 366 estimates and uncertainties of the parameters, given the 367 data and assuming one specific model.

368 Once the posterior distribution of all model parameters 369 is so obtained, we can compute the distribution of the
370 excess loss angle for each resonant mode and compare 371 the results with the experimental measurements. This is 372 done by using each point in the parameter space obtained 373 from the MCMC sampler in the corresponding model to 374 compute the excess loss, and then producing a histogram 375 of all values. Figure 2 shows the results for both model 376 considered here as an example: single loss angle with lin377 ear frequency dependency and different bulk shear loss 378 angles, again with linear frequency dependency (similar 379 results for all annealing temperatures and power law fre380 quency dependency are also available, but not shown here 381 for brevity). In those plots the distribution of the excess 382 loss angles are shown and compared with the experimen383 tal results. In the case of the bulk and shear loss angle 384 model, both contributions are shown separately, together 385 with the sum. One can notice that most of the excess loss 386 angle is due to the shear contribution, but there is nev387 ertheless a not negligible contribution coming from the 388 bulk losses.

${ }_{389}$ The Bayesian approach we used to fit the model parame390 ters allows us to compute the probability of the different 391 models $\mathcal{P}\left(\mathcal{M}_{j} \mid \delta \phi_{i}\right)$, given the measured data points. Us392 ing Bayes' theorem again, this can be written as

$$
\mathcal{P}\left(\mathcal{M}_{j} \mid \delta \phi_{i}\right)=\frac{\mathcal{P}\left(\delta \phi_{i} \mid \mathcal{M}_{j}\right) \mathcal{P}\left(\mathcal{M}_{j}\right)}{\mathcal{P}\left(\delta \phi_{i}\right)}
$$

${ }^{393}$ where $\mathcal{P}\left(\mathcal{M}_{j}\right)$ is the prior probability of the models, and ${ }_{394} \mathcal{P}\left(\delta \phi_{i} \mid \mathcal{M}_{j}\right)$ is the likelihood of obtaining the measured 395 data points given the model. The latter can be computed 396 from the results of the MCMC sampler as explained ${ }^{397}$ above. The term in the denominator acts as a normal398 ization constant, independent of the model. Therefore, 399 assuming all models are equally likely a priori, we can 400 compute the logarithm of the Bayesian odd ratio of any 401 pair of models, given the data:

$$
\log O\left(\mathcal{M}_{1}, \mathcal{M}_{2}\right)=\log \left[\frac{P\left(\mathcal{M}_{1} \mid \delta \phi_{i}\right)}{P\left(\mathcal{M}_{2} \mid \delta \phi_{i}\right)}\right]
$$

${ }_{402} \mathrm{~A}$ logarithm odd ratio greater than zero means that the 403 measured data favors the model at the numerator $\mathcal{M}_{1}$, 


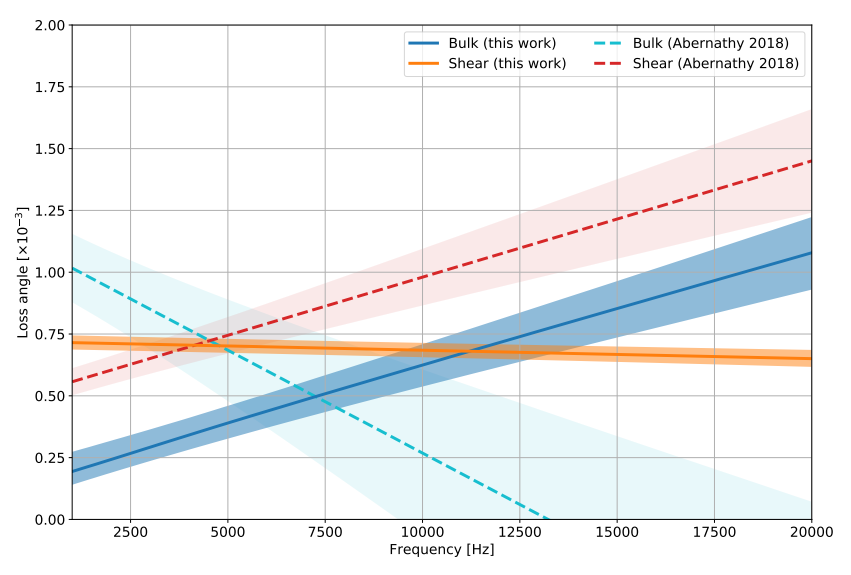

FIG. 4. Comparison of bulk and shear loss angles for the as deposited titania-doped tantala, as obtained in this work and as reported in Abernathy et al. [18].

404 while a value lower than zero means that the model at 405 the denominator $\mathcal{M}_{2}$ is favored. We use the Bayesian 406 odd ratios to determine which model is favored by the 407 data, since this approach takes naturally into account 408 the uncertainty in the data points and in the estimated 409 film mechanical properties, as well as the different dimen410 sionality of the parameter space for each model. It also ${ }_{411}$ provides a quantitative measurement of the "goodness of 412 the fit" based on the model complexity and measurement 413 uncertainties.

${ }_{414}$ Table II lists the logarithm of the odd ratio for pairs 415 of models. For all the annealing temperature, as well 416 as for the as deposited film, the measured data strongly ${ }_{417}$ favor the models with different bulk and shear loss angles. ${ }_{418}$ Among those models, the linear frequency dependency ${ }_{419}$ is slightly favored. Table III summarizes the estimated ${ }_{420}$ parameters for this model. Figure 3 shows the results in ${ }^{421}$ graphical form. In the same plot we compare the bulk ${ }_{422}$ and shear loss angles with the estimate obtained using a ${ }_{423}$ single loss angle model, as done in most of previous work. ${ }_{424}$ Figure 4 compares our results for the as-deposited film 425 with those reported in Abernathy et al. [18], where a ${ }_{426}$ similar analysis was performed. Our results are not con427 sistent with those reported in that work, showing oppo${ }_{428}$ site frequency dependencies and different relative ampli429 tude of the two loss angles. We should note that the 430 two films, although both being made of about $20 \%$ ti431 tania doped tantala, were produced by different groups ${ }_{432}$ employing different coating deposition chambers (in our 433 case, films were grown by reactive ion beam sputtering 434 using the Laboratory Alloy and Nanolayer System man435 ufactured by 4Wave, Inc [19] at Colorado State Univer${ }_{436}$ sity; in Abernathy's case, an ion beam sputtering sys${ }_{437}$ tem was used by the Commonwealth Scientific and In438 dustrial Research Organization [20]) and therefore might 439 have different properties. If we assume that the two films ${ }_{440}$ have similar properties, the reason for the discrepancy ${ }_{441}$ is not understood at the moment of writing. However, 442 we would like to point out some key differences between
${ }_{443}$ the measurement reported in Abernathy et al. [18] and 444 our results: the samples were suspended with different 445 techniques, which might induce systematic differences; 446 we measured and subtracted the contribution to the loss 447 angle of the uncoated substrate, while it is not clear how ${ }_{448}$ that was treated in Abernathy's work; in our work a 449 larger number of resonant mode was probed; in Aber450 nathy's work bulk and shear loss angles are extracted ${ }_{451}$ from pairs of Q measurement, assuming no frequency de${ }_{452}$ pendency between the two modes in each pair but allow${ }_{453}$ ing for a frequency dependency between pairs, while in 454 our work we directly fit a frequency dependent model to 455 the experimental data; finally, in our work we restricted ${ }_{456}$ the fit parameters to physically realizable values, while ${ }_{457}$ in Abernathy's the bulk loss angle is predicted to have 458 negative values for high frequencies.

${ }_{459}$ In this analysis the film is assumed to have uniform 460 thickness and mechanical properties, and to cover the ${ }_{461}$ entire substrate surface. The expected variation of the ${ }_{462}$ film thickness over the surface is expected to be small. ${ }_{463}$ However, variations of the film properties with position 464 might introduce mode-dependent systematic errors that 465 have not been considered in this study. Further work is 466 needed to quantify their effect on the bulk and shear loss 467 angle results.

${ }_{468}$ In previous works [21], the mechanical quality factors of 469 uncoated silica disks were found to be dependent and 470 limited by loss mechanisms at the unpolished edge, and 471 were also found to degrade over time. The silica disks 472 used in this work have an optical quality polished edge, 473 and the mechanical quality factors have been measured 474 before the film deposition, to ensure a correct subtraction 475 of the background due to the substrate. We also verified 476 that the polished edge ensures that there is no signifi477 cant evolution of the substrate quality factor over time. ${ }_{478}$ Therefore we are confident that the effect described in $479[21]$ is not an issue in our work.

\section{${ }_{480}$ IV. EFFECT ON THERMAL NOISE ESTIMATE}

${ }_{481}$ The standard computations used to estimate the contri${ }_{482}$ bution of coating thermal noise in the advanced gravita483 tional wave detectors [5] assume that both the low and 484 high index materials can be described with one single loss 485 angle. Direct thermal noise measurements have also been 486 performed [28] and the results expressed again in terms of ${ }_{487}$ equal bulk and shear loss angles. Here we use the result of 488 our analysis, and compute the expected thermal noise for 489 a high reflectivity mirror similar to the design employed 490 in the Advanced LIGO detectors, using the inferred bulk 491 and shear loss angles. We use the model described in ${ }_{492}$ Hong et al. [13] (in particular starting from equation 94 493 therein), where the properties of the component materi494 als and the geometry of the layers are used to predict the 495 total thermal noise. Possible effects due to the transition 496 between layers are not considered [6, 29].

${ }_{497}$ We consider a high reflection coating composed of 38 al498 ternating layers of silica (low index material) and titania- 


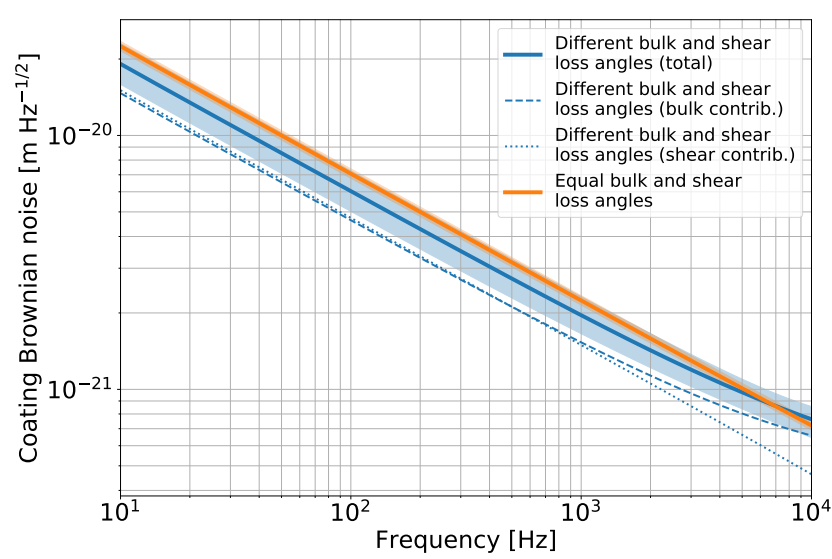

FIG. 5. Brownian noise for a single high reflectivity mirror, composed of alternating layers of silica and titania-doped tantala, as described in the main text. The solid orange line shows the displacement noise using the model where the bulk and shear loss angles are equal, while the solid blue line corresponds to the model where bulk and shear can assume different values. The dashed and dotted curves show the bulk and shear contribution, respectively.

499 doped tantala (high index material), each with an optical ${ }_{500}$ thickness of $\lambda / 4$ where the laser wave-length $\lambda$ in vacuum 501 is $1064 \mathrm{~nm}$, to obtain a nominal transmission of about 5 $502 \mathrm{ppm}$ [30]. For the titania-doped tantala loss angle we ${ }_{503}$ use the results reported in this work, for the film mea504 sured after annealing at $500^{\circ} \mathrm{C}$. We compare two differ505 ent cases: the best fit to a single loss angle and the best 506 fit with different bulk and shear loss angles, as shown ${ }_{507}$ in figure 3. The contribution of silica to thermal noise 508 is small, but nevertheless we included a frequency de${ }_{509}$ pendent model obtained from another measurement we 510 performed on silica thin films annealed at $500^{\circ} \mathrm{C}$. In this 511 case the sensitivity of our ring-down measurement was 512 not enough to disentangle bulk and shear loss angles: 513 the experimental data is best described by a single loss 514 angle, linearly dependent on the frequency, given by

$$
\begin{aligned}
\phi_{\mathrm{SiO}_{2}}(f)= & (0.035 \pm 0.004) \times 10^{-3} . \\
& {\left[1+(-0.006 \pm 0.007) \times 10^{-3} \frac{f-1 \mathrm{kHz}}{1 \mathrm{kHz}}\right] }
\end{aligned}
$$

${ }_{515}$ Figure 5 shows the displacement noise due to the Brown516 ian noise of a single high reflectivity mirror. As a ref${ }_{517}$ erence, assuming the best fit to the data with a sin518 gle loss angle, we obtain a coating Brownian noise of ${ }_{519}(7.0 \pm 0.3) \times 10^{-21} \mathrm{~m} / \sqrt{\mathrm{Hz}}$ at $100 \mathrm{~Hz}$. Using instead
${ }_{520}$ the best fit to the data with different bulk and shear ${ }_{521}$ loss angles, we obtain $(6.0 \pm 1.1) \times 10^{-21} \mathrm{~m} / \sqrt{\mathrm{Hz}}$ at 100 ${ }_{522} \mathrm{~Hz}$. For comparison, the direct thermal noise measure523 ment reported in [28] can be extrapolated to a level of ${ }_{524}(7.5 \pm 0.1) \times 10^{-21} \mathrm{~m} / \sqrt{\mathrm{Hz}}$ at $100 \mathrm{~Hz}$. Within the preci525 sion of our measurement, there is no significant impact on 526 the estimate of thermal noise for and Advanced-LIGO527 like high reflectivity coating.

${ }_{528}$ It is worth noting that the knowledge of the separate bulk 529 and shear loss angles could allow an additional degree of 530 freedom to optimize the thermal noise of the coating, by ${ }_{531}$ changing the thickness of the layers [13].

\section{CONCLUSIONS}

${ }_{533}$ We showed that it is possible to estimate the bulk and 534 shear contribution to the loss angle of a thin film, using 535 measurements of the decay time of the resonant modes 536 of a coated silica disk, carried out in a Gentle Nodal ${ }_{537}$ Suspension system. As an example we analyzed a thin ${ }_{538}$ film of titania-doped tantala, one of the materials used 539 in the advanced gravitational wave interferometric detec540 tor mirrors. A Bayesian analysis of the experimental data ${ }_{541}$ shows that a model featuring different bulk and shear loss ${ }_{542}$ angle is favored with respect to a simpler model with one 543 single loss angle (i.e. same loss angle for bulk and shear 544 energies). The change in loss angles with annealing is 545 more evident in the shear than in the bulk contribution. ${ }_{546}$ When the two models are used to compute the expected 547 thermal noise for a high reflection mirror similar to those 548 used in Advanced LIGO, the difference is marginal and 549 within error bars when the measurements are extrapo550 lated in the frequency region between 10 and $1000 \mathrm{~Hz}$.

\section{ACKNOWLEDGMENTS}

${ }_{552}$ LIGO was constructed by the California Institute of ${ }_{553}$ Technology and Massachusetts Institute of Technology 554 with funding from the United States National Science 555 Foundation under grant PHY-0757058. This work was 556 also supported by the Center for Coating Research, NSF 557 grants PHY-1708010 PHY-1710957 and by the LIGO 558 Laboratory, NSF grant PHY-1764464. The authors are ${ }_{559}$ grateful for computational resources provided by the 560 LIGO Laboratory and supported by the National Science ${ }_{561}$ Foundation Grants PHY-0757058 and PHY-0823459. ${ }_{562}$ This paper has LIGO document number P1900336.
1] J Aasi and others [LIGO Scientific Collaboration]. Ad- 570 vanced LIGO. Class. Quantum Grav., 32:074001, 2015. 571

[2] B. Willke, B. et al. The GEO 600 gravitational wave 572 detector. Class. Quantum Grav. 19, 1377-1387 (2002) 573

3] Somiya. K and others [KAGRA collaboration]. Detec- 574 tor configuration of KAGRA - the Japanese cryogenic 575 gravitational-wave detector. Class. Quantum Grav., ${ }^{576}$
29:124007, 2011.

[4] F Acernese and others [Virgo Collaboration]. Advanced Virgo: a 2nd generation interferometric gravitational wave detector. Class. Quantum Grav., 32:024001, 2015.

[5] Harry, G.M., Gretarsson, A.M., Saulson, P.R., and others. Thermal noise in interferometric gravitational wave detectors due to dielectric optical coatings. Classical and 
Quantum Gravity, 19(5), (2002) p.897

6] M. Granata, et al. Amorphous optical coatings of present 612 gravitational-wave interferometers arXiv:1909.03737 61 [physics.ins-det]

7] H.B. Callen and T.A. Welton Irreversibility and Gener- 615 alized Noise Physical Review. 83: 34 ?40 (1951) 616

[8] Y. Levin Internal thermal noise in the LIGO test masses: 617 A direct approach Phys. Rev. D 57, 659 (1998)

[9] P. Stoica and R. Moses Spectral Analysis of Signals Prentice Hall, Upper Saddle River, New Jersey (2005)

10] S. Reid, I. W. Martin Development of Mirror Coatings for Gravitational Wave Detectors Coatings 6, 61 (2016) ${ }_{622}$

11] W. A. Phillips, Tunneling States in Amorphous Solids 6 Journal of Low Temperature Physics 7, 351 (1972)

12] K. A. Topp and D. G. Cahill Elastic properties of several 625 amorphous solids and disordered crystals below $100 \mathrm{~K} \mathrm{Z}$. Phys. B 101, 235(1996)

[13] T. Hong, et al. Brownian thermal noise in multilayer coated mirrors Phys. Rev. D 87, 082001 (2013)

4] T. Li, et al. Measurement of mechanical thermal noise and energy dissipation in optical dielectric coatings Phys. Rev. D 89, 092004 (2014)

15] E. Cesarini et al. A gentle nodal suspension for measurements of the acoustic attenuation in materials. Rev. Sci. ${ }^{634}$ Instrum., 80, 053904 (2009)

16] G Vajente et al. A high throughput instrument to measure mechanical losses in thin film coatings Rev. Sci. 637 Instrum., 88:073901, (2017)

17] H.-W. Pan, et al. Silicon nitride and silica quarter-wave stacks for low-thermal-noise mirror coatings Phys. Rev. ${ }^{64}$ D 98, 102001 (2018)

[18] M. Abernathy et al., Bulk and shear mechanical loss of 64 titania-doped tantala Physics Letters A, 382, 2282-2288 (2018)
1 [19] M. Fazio, L. Yang, A. Markosyan, R. Bassiri, M. M. Fejer and C. S. Menoni Growth and characterization of Sc2O3 doped Ta2O5 thin films Appl. Opt. 59, A106 (2020)

[20] R. P. Netterfield, M. Gross, F. N. Baynes, K. L. Green, et al. Low mechanical loss coatings for LIGO optics: progress report. In M. L. Fulton and J. D. T. Kruschwitz, editors, Society of Photo-Optical Instrumentation Engineers (SPIE) Conference Series, volume 5870, 144 (2005)

[21] G. Cagnoli et al. Mode-dependent mechanical losses in disc resonators, Phys. Lett. A 382 (2018) 2165

[22] A. Gelamn, J. B. Carlin, H. S. Stern, D. B. Dunson, A. Vehtari, D. B. Rubin Bayesian Data Analysis, 3rd edition Chapman and Hall/CRC (2013)

24 [23] D. Silvia and J. Skilling Data Analysis: a Bayesian Tutorial Oxford University Press Oxford (2006)

626 [24] U. von Toussaint Bayesian inference in physics Rev. Mod. Phys. 83, 943 (2011)

[25] M. Bischoff et al. Postdeposition treatment of IBS coatings for UV applications with optimized thin-film stress properties Applied Optics 53, A212-A220 (2014)

[26] J. T. Brown Center wavelength shift dependence on substrate coefficient of thermal expansion for optical thinfilm interference filters deposited by ion-beam sputtering Applied Optics 43, 4506-4511 (2004)

635 [27] D. Foreman-Mackey, D. W. Hogg, D. Lang and J. Goodman emcee: The MCMC Hammer arXiv:1202.3665 [astro-ph.IM]

638 [28] S. Gras and M. Evans, Direct measurement of coating thermal noise in optical resonators Phys. Rev. D 98, 122001

641 [29] M. Granata et al. Mechanical loss in state-of-the-art amorphous optical coatings Phys. Rev. D 93, 012007 (2016)

644 [30] A. Amato et al., High-Reflection Coatings for Gravitational-Wave Detectors: State of The Art and Future Developments J. Phys.: Conf. Ser. 957012006 (2018) 